\title{
Enhancing the Quality of Service in MANETs by Improving the Routing Techniques
}

\author{
Mamatha Balachandra \\ Department of Computer \\ Science and Engineering, \\ Manipal Institute of Technology, \\ Manipal University, India
}

\author{
Prema K V \\ FET, \\ Department of Computer \\ Science and Engineering, \\ MITS University, \\ Rajasthan, India
}

\author{
Krishnamoorthy M \\ Department of Master of \\ Computer Applications, \\ Manipal Institute of \\ Technology, Manipal \\ University, India
}

\begin{abstract}
The aim of this work is to modify the existing MANET reactive Multipath routing protocol Ad hoc On-demand Distance Vector (AOMDV). Since the Route discovery and route maintenance of AOMDV Routing $25 \mid \mathrm{P}$ a g e protocol lead to a high number of unsuccessful packet deliveries from the source nodes to the destination nodes during broadcasting, it results in reducing the quality of service support for Mobile Ad Hoc Networks. This paper is about the systematic performance study made of existing routing protocol for Ad Hoc networks AOMDV based on QoS parameters delay and packet delivery ratio. Further, a novel Multipath QoS Aware Routing Protocol (MQARP) based on AOMDV is proposed to support delay, jitter and throughput constraints. Simulation using various traffic sources and movement patterns is made and existing version of the protocol is modified. The QoS metrics are measured by varying the pause time, speed and number of nodes. The performance of AOMDV and MQARP are compared using the network simulator. The simulator NS2.34 is used to simulate the performance of the metrics.
\end{abstract}

\section{General Terms}

Mobile Ad Hoc Networks, Quality of Service

\section{Keywords}

MANET, QoS, AOMDV, MQARP, throughput, jitter, delay.

\section{INTRODUCTION}

Recent progress in mobile wireless networking has provided a major impetus toward the development of self-created, selforganized[1] and rapidly deployable network architecture referred to as Mobile Ad Hoc network. The growth of real time applications in such a environments have drawn a lot of attention to wireless networks that support quality of service (QoS)[2][3]. Many different protocols have been proposed to support QoS in MANETs, each based on different assumptions and intuitions.

\subsection{AOMDV Routing}

AOMDV (Ad Hoc On-demand Multipath Distance Vector) is a multi-path routing protocol [4]. It is an extension to AODV[5] and provides two main services i.e. route discovery and maintenance. Unlike AODV, every RREP is being considered by the source node and thus multiple paths can be discovered in one route discovery. Being the hop-by-hop routing protocol, the intermediate node can maintain multiple path entries in their respective routing table.

AOMDV is considered more efficient in terms of creating less overhead Number of paths in any given source and destination is directly proportional to the number of nodes in entire network. AOMDV works more efficiently in dense and heavy networks. Fig. 1 is the Route discovery in Ad hoc On-demand Multipath Routing:

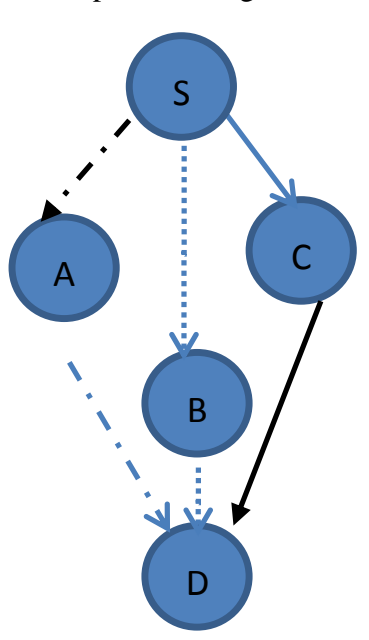

(a) 3 Node disjoint paths SAD,

SBD and SCD

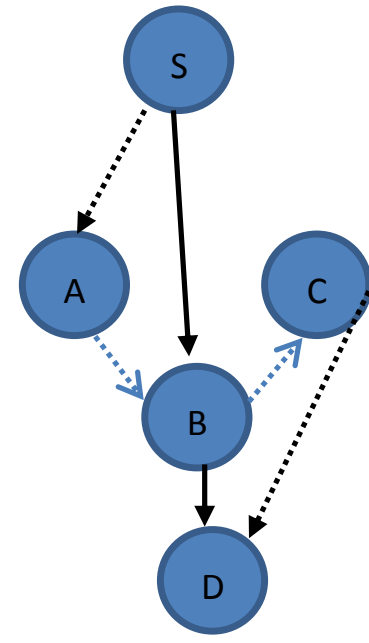

(b) 2 Link disjoint $S B D$ and $S A B C D$
Fig. 1 AOMDV Multi-path Routing

AOMDV is an on-demand routing protocol that builds multiple routes using request/reply cycles. When the source needs a route to the destination but no route information is known, it floods the ROUTE REQUEST (RREQ) message to the entire network. Because this packet is flooded, several duplicates that traversed through different routes reach the destination. The destination node selects multiple disjoint routes and sends ROUTE REPLY (RREP) packets back to the source via the chosen routes. The purpose of computing alternate paths for a source node is that when the primary path breaks due node movement, one of the alternate paths can then be chosen as the next primary path and data transmission can continue without initiating another route discovery.

To discover distinct paths, AOMDV suppresses duplicate route requests (RREQs) at intermediate nodes. Such suppression comes in two different variations, resulting in either node (illustrated in Fig. 1 (a)) or link (illustrated in Fig. 1(b)) disjoint. AOMDV can be configured to either discover the link (no common link between any given pair of nodes) or node (in addition to link disjoint, common intermediate nodes are also excluded between any given pair of nodes) disjoints paths. 


\subsection{Quality of Service}

QoS is usually defined as a set of service requirements that needs to be met by the network while transporting a packet stream from a source to its destination. The network needs are governed by the service requirements of end user applications. The network is expected to guarantee a set of measurable prespecified service attributes to the users in terms of end-to-end performance, such as delay, bandwidth, probability of packet loss, delay variance (jitter), etc. Power consumption is another QoS attribute which is more specific to MANETs [2]. Providing QoS support in MANETs is an active research area. MANETs have certain unique characteristics that pose several difficulties in provisioning QoS.

\section{RELATED WORK}

Numerous QoS routing protocols have been proposed for wireless ad hoc networks. Many of them are based on the popular on-demand routing protocols, DSR and AODV. Following papers highlights main points about the work that took place in that area.

(a) Shahram Jamali, Bita Safarzadeh, Hamed Alimohammadi, "A stable QoS aware reliable on-demand distance vector routing protocols for mobile Ad Hoc networks", Scientific Research and Essays Volume 6, Academic Journals , July 2011[6]. This paper highlights the following significant points:

Recently, many routing protocols were proposed for MANETs that use global positioning system (GPS). The coordinates of each node can be known using GPS. Further, the transmission routing protocols can complete the process of route discovery by mathematically calculating the routing. Due to the mobility of mobile nodes in MANETs, the shortest path is not necessarily the best path. If we do not consider the stability of routing paths, then wireless links may be easily broken. There have been many efforts made to design a reliable routing protocol to enhance a network's stability. In order to select a reliable route proposed protocol uses 3 parameters: route life time, mobility and number of hops.

(b) S. Chakrabarti and A. Mishra, "Quality of Service Challenges for Wireless Mobile Ad Hoc Networks," International Journal of Wireless Communication and Mobile Computing, Volume 4, pp. 129-53, March 2004 [7] .Their conclusions highlighted several significant points :

Many of the underlying algorithmic problems, such as multiconstraint routing, have been shown to be NP-complete. QoS and, indeed, best-effort routing can only be successfully achieved if the network is combinatorially stable. This means that the nodes are not moving faster than routing updates can propagate. Different techniques are required for QoS provisioning when the network size becomes very large, since QoS state updates would take a relatively long time to propagate to distant nodes. There is a trade-off between QoS provisioning and minimization of power utilization.

(c) J. Stine and G. de Veciana, "A Paradigm for Quality of Service in Wireless Ad Hoc Networks using Synchronous Signaling and Node States," IEEE Journal of Selected Areas in Communications, Volume 22, Sept. 2004, pp. 1301-21 [8]. This paper highlights several significant points:

A major advantage of discovering QoS state proactively surfaces in situations where different applications specify their requirements with different metrics. As long as it is decided which QoS states to keep up-to-date, a route may be computed from the routing table based on any QoS metric, without the need for a separate discovery process for each metric. A purely reactive routing solution avoids the potential wastage of channel capacity and energy by discovering QoS routes.

(d) TaejoonPark, Student Member, IEEE, and Kang G. Shin, Fellow, IEEE, "Optimal Tradeoffs for Location-Based Routing in Large-Scale Ad Hoc Networks", 2005, IEEE TRANSACTIONS ON NETWORKING, VOL. 13, NO. 2, APRIL [9].

This paper states that while simple multi-constraint QoS routing proposals are numerous, there are few that attempt to optimize multi-constraint routing. One example was based on genetic algorithms. However, such methods have limited applicability due to the overhead and energy cost of collecting enough state information. Accurate studies are required to establish, with various networking environments and topologies, whether or not it is feasible to collect and maintain sufficient state information to apply methods such as GAs. For the cases where it is, more research is required on different types of heuristic algorithms for calculating near optimal paths with multiple QoS constraints. Comparative studies on the performance and impact of the heuristics, are additional future work.

(e) Ronald Beaubrun and BadjiMolo, "Using DSR for Routing multimedia traffic in MANETs", January 2010 [10]:

This paper discusses an extension of the on-demand DSR protocol. It consists of a scheme to distribute traffic among multiple routes in a network. Its performance in terms of delay degrades (reaches to 2.2 Seconds) as the traffic increases i,e. 40 and above.

(f) Chandra Mouli Venkata Srinivas Akana Sandeep Kumar, Dr C Divakar "QoS for Real time transmission on MANETs", International Journal of Advanced Networking and Applications volume: 02, Issue: 03, Pages: 679-685 (2010)[11]:

This paper states that for a QoS AODV routing protocol, problems would rise when the node density of the network is high. The reason is that the QoS AODV routing protocol uses the control message to exchange information between neighbors. When the node density is too high, the sending of control will cost much available data rate. As a result, the network will be ruined and traffic will be delayed more since control messages have higher priority than data packets. To conclude, it is predicted that the QOS AODV will not work well in high density ad hoc networks.

\section{MULTIPATH QOS AWARE ROUTING PROTOCOL (MQARP)}

\subsection{Overview}

Multipath QoS Aware Routing Protocol (MQARP) is a linkdisjoint on-demand multipath routing protocol which aims to identify more than one routes which are link reliable and delay aware. The metrics used for finding the QoS routes are "Average timestamp" and the "Link life time ratio". Since EMQARP is a multipath routing protocol it finds multiple paths from source to destination in a single discovery. Once the route is discovered, every node on the route calculates average delay and the link life time ratio. If the computed values exceeds a predetermined threshold, it sends a notification to the sender. Also from that node the further broadcasting of RREQ message is avoided if the timestamp is more and link life time is less. Then the sender switches to the next Quality of Service Path available in the routing table. If there exists no path, route discovery phase is reinitiated. 


\subsection{MQARP Route discovery}

Route discovery phase of MQARP is same as AOMDV routing protocol which is initiated when a source node wants to send data to another node for which it does not have routing information or when current active path for destination fails. During the route discovery of the MQARP, it broadcasts route request (RREQ) packets to its neighbor containing the following information:

$\begin{array}{lll}\text { (i) } & \text { Source address } & \text { (ii) Source Sequence Number } \\ \text { (iii) } & \text { Broadcast ID } & \text { (iv) Destination address } \\ \text { (v) } & \text { Destination Sequence Number } \\ \text { (vi) } & \text { Hop count } & \text { (vii) Time stamp and } \\ \text { (viii) } & \text { Link life time } & \end{array}$

While broadcasting RREQ packets every node in the network will save the following information in terms of RREP message, so as to establish a reverse path from destination to source:
(i) Source address
(ii) Source Sequence Number

(iii) Broadcast ID (iv) Destination address

(v) Minimum link life time

The reverse path is used to forward RREP packets from the destination to the source. The intermediate node drops the RREQ packets either if the Time stamp is more than the threshold value or if it is the duplicate RREQ. On receiving the RREP every intermediate node updates the following routing table entries:
a. Destination address
b. Next hop
c. Hop count
d. Destination Sequence Number
e. Percentage Link life time and
f. Average Time stamp

\subsection{Calculating Average Time stamp}

In the new QoS Routing Protocol, the loss of unnecessary packet is avoided. Each of the packets broadcasted by the source node across the network has a timestamp associated with it. As the nodes are updated in the routing table, we calculate the average timestamp value using the following equation :

$$
\mathbf{T}_{\mathrm{avg}}=\frac{\sum_{i=0}^{i=n} T i}{C}
$$

Where Tavg is the Average Timestamp, $\mathrm{n}$ stands for maximum simulation time, $\mathrm{Ti}$ is the Timestamp of each Packet and $\mathrm{C}$ is the Total count of each entry made to the Routing table. This average is a runtime average which is directly proportional to the number of nodes $\mathrm{N}$. As the number of nodes increase, the number of nodes getting added to the Routing Table also increases:

$$
\text { Tavg } \alpha \mathrm{N}
$$

The positions of the node added to the Routing table is known. Henceforth, if there is a particular node which is very far away such that its timestamp is higher than that of the average value, re-broadcasting of the RREQ from that node is not allowed. In this way, we save the loss of packets and force the Route Discovery Process to search for another route with limited time.

\subsection{Calculation of Percentage Life Time Ratio (PLTR)}

Due to dynamic change in topology of the Ad Hoc network, it is required to compute the route reliability dynamically.

Assuming two mobile nodes A and B are within the radio transmission range of each other, let:

$\left(\mathrm{X}_{\mathrm{A}}, \mathrm{Y}_{\mathrm{A}}\right)$ : coordinate of mobile node $\mathrm{A}$;

$\left(\mathrm{X}_{\mathrm{B}}, \mathrm{Y}_{\mathrm{B}}\right)$ : coordinate of mobile node $\mathrm{B}$;

$\mathrm{V}_{\mathrm{A}}$ : mobility speed of mobile node $\mathrm{A}$;

$\mathrm{V}_{\mathrm{B}}$ : mobility speed of mobile node $\mathrm{B}$;

$\Theta_{A}$ : direction of motion of mobile node $\mathrm{A}(0<\Theta \mathrm{A}<2 \pi)$;

$\Theta_{\mathrm{B}}$ : direction of motion of mobile node $\mathrm{B}(0<\Theta \mathrm{B}<2 \pi)$.

Using the aforementioned parameters, we can define the link life time equation as follows :

$$
\mathrm{LLT}=\frac{-(a b+c d)+\sqrt{\left(a^{2}+c^{2}\right) r^{2}-(a d-c b)^{2}}}{\left(a^{2}+c^{2}\right)}
$$

Where,

$$
\begin{array}{ll}
\mathrm{a}=\mathrm{V}_{\mathrm{A}} \cos \Theta_{\mathrm{A}}-\mathrm{V}_{\mathrm{B}} \cos \Theta_{\mathrm{B}}, & \mathrm{c}=\mathrm{V}_{\mathrm{A}} \sin \Theta_{\mathrm{A}}-\mathrm{V}_{\mathrm{B}} \sin \\
\Theta_{\mathrm{B}} & \mathrm{d}=\mathrm{Y}_{\mathrm{A}}-\mathrm{Y}_{\mathrm{B}}
\end{array}
$$

The link life time is calculated at each hop during the route request packet is traversing the path. Each node calculates the life time of the link between itself and previous hop. If node A is the previous hop of the packet for node $\mathrm{B}$, it appends its position and movement information to the route request packet. When node B receives this packet, it calculates the life time of the link. The Route Life Time (RLT) [11] is the minimum link life time along a routing path. Therefore, the RLT is equal to the minimum of LLTs for a route.

The formula to compute PLTR is as shown below:

$$
\mathrm{PLTR}=\frac{\text { Route LifeTime }}{\text { TTL }} * 100
$$

Where TTL carries a time to live (TTL) value that states for how many hops this message should be forwarded. This value is set to a predefined value at the first transmission and increased at retransmissions. Retransmissions occur if no replies are received. The LTR multiplied by 100 gives the Percentage Life Time Ratio (PLTR) for a route. If the PLTR is bigger than $50 \%$ then the intermediate node allows the rebroadcasting of RREQ messages.

Multipath QoS Aware Routing Protocol (MQARP) is a link-disjoint on- demand multipath routing protocol which aims to identify more than one routes which are link reliable and delay aware. The metrics used for finding the QoS routes are "Average timestamp" and the "Link life time ratio". Since MQARP is a multipath routing protocol it finds multiple paths from source to destination in a single discovery. Once the route is discovered, every node on the route calculates average delay and the link life time ratio. If the computed values exceeds predetermined threshold, it sends a notification to the sender. Also from that node the further broadcasting of RREQ message is avoided if the timestamp is more and link life time is less. Then the sender switches to the next Quality of Service Path available in the routing table. If there exists no path, route discovery phase is reinitiated. 


\section{SIMULATION SETUP}

The NS-2.34 [12][13] is used for simulation. It has support for simulating multi hop wireless networks. We simulated numerous test conditions using CBR traffic.

\subsection{Movement Model}

The mobile nodes move according to the random waypoint model. Each mobile node begins the simulation by remaining stationary for pause time seconds. It then selects a random destination in the defined topology area and moves to that destination at a random speed. The random speed is distributed uniformly between zero (zero not included) and some maximum speed. Upon reaching the destination, the mobile node pauses again for pause time seconds, selects another destination, and proceeds.

\subsection{Communication Model}

In the scenario used in this study, up to 150 nodes are generated and the traffic connection pattern is generated by cbrgen.tcl. The Table 1 shows the simulation parameters:

Table 1: Simulation parameters

\begin{tabular}{|l|l|}
\hline Simulation time & 200 seconds \\
\hline Number of nodes & $10,20,30,40,60,80,100,120,140,150$ \\
\hline Map size & 1000 X 1000 \\
\hline Speed & $5 \mathrm{~m} / \mathrm{sec}, 15 \mathrm{~m} / \mathrm{sec}, 25 \mathrm{~m} / \mathrm{sec}$ \\
\hline Mobility Model & Random Way Point \\
\hline Traffic type & CBR \\
\hline Packet size & 512 bytes \\
\hline Pause time & $0,10,20,40,60,80,100$ \\
\hline
\end{tabular}

\subsection{Simulation Parameters}

The NS-2.34 is used for simulation. It has support for simulating multi hop wireless networks. We simulated numerous test conditions using CBR traffic. The simulation is run using various scenarios (such as varying the pause time and speed) and traffic patterns (such as varying the number of nodes). To overcome the effect of randomness in the output we have taken the averages of the results to get their realistic values. Simulations are carried out by varying the pause time, speed and node density simultaneously. The simulation results reveal some important characteristic differences between the Following metrics are used to compare the performances of two routing protocols:

Throughput: The rate at which bulk data transfers can be transmitted from one host to another over a sufficiently long period of time and is measured in Kbits/sec.

Throughput $=$ Packet length $/$ time in seconds

Delay: End-to-end delay indicates how long it took for a packet to travel from the application layer of the source to the application layer of the destination and is measured in milliseconds

Jitter: It is a parameter that measures the change in network latency over a short periods of time. There are several ways to measure this parameter including measuring the difference in

Latency between consecutive packets or measuring packets transmitted within a specified time interval. Jitter particularly affects the performance of real time network applications such as streaming video and audio. It is measured in milliseconds.

\subsection{Simulation Results}

The base protocol used to compare the performance of MQARP is the AOMDV [11]. The metrics used in comparing these two protocols are Throughput, Jitter and End-to-End delay.

4.4.1 Throughput versus Mobility and Number of nodes The Figure 2 shows the Throughput versus pause time for the number of nodes $=80$. And Figure 3 shows Throughput versus Number of nodes for the Pause time $=10$ seconds

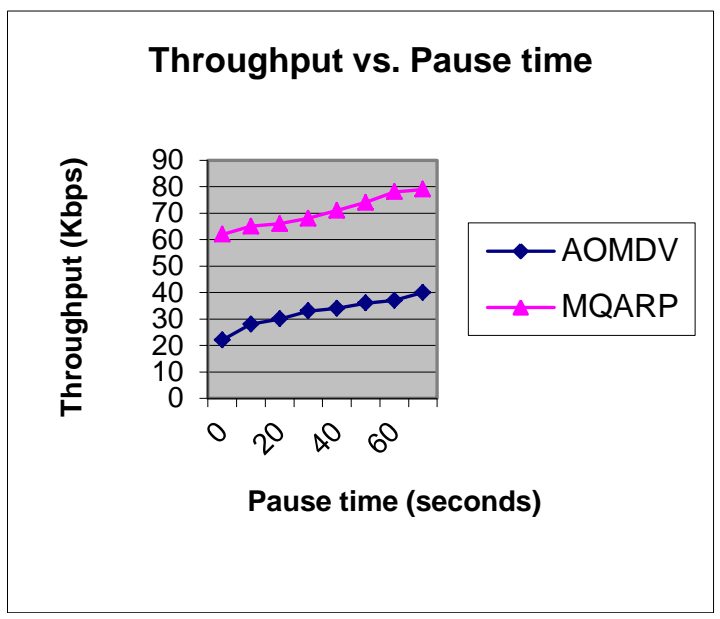

Figure 2 Throughput versus Pause time Throughput vs. No. of Nodes

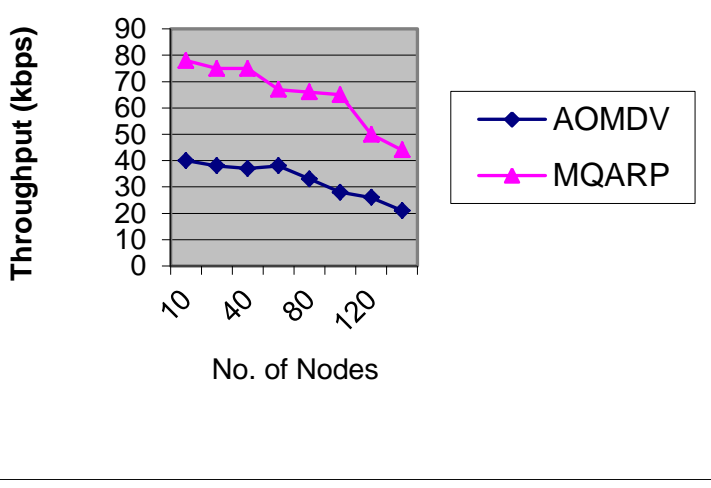

Figure 3 Throughput versus No. of Nodes

From the figure 2 it can be observed that for the low pause time the throughput is less. That means for high mobility the performance of the protocols in terms of the throughput degrades. Similarly the figure 3 shows that as the number of nodes are increasing the performance in terms of throughput degrades. But in both the cases the throughput of the MQARP is better compared the AOMDV protocol.

4.4.2 Delay versus Mobility and Number of nodes

The Figure 4 shows the Delay versus pause time for the number of nodes $=80$. And Figure 5 shows Delay versus Number of nodes for the Pause time $=10$ seconds 


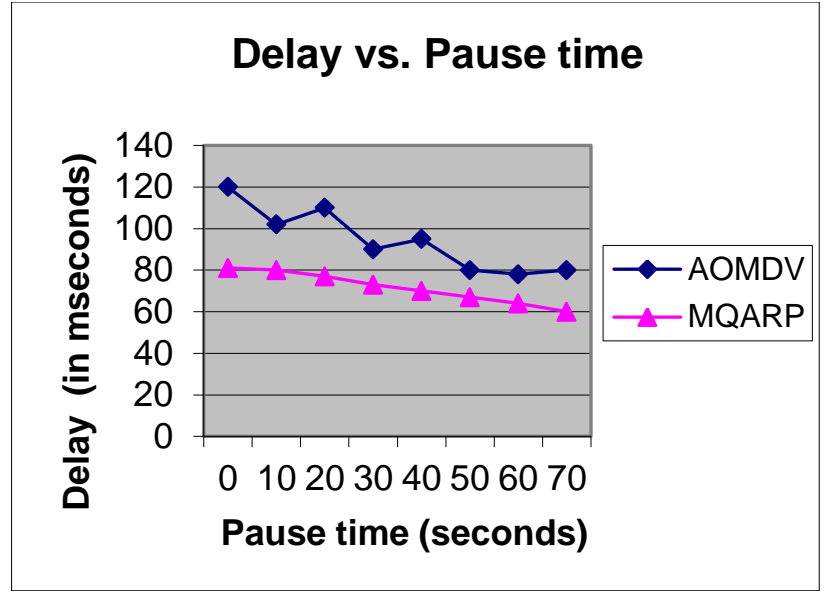

Figure 4 Delay versus Pause time

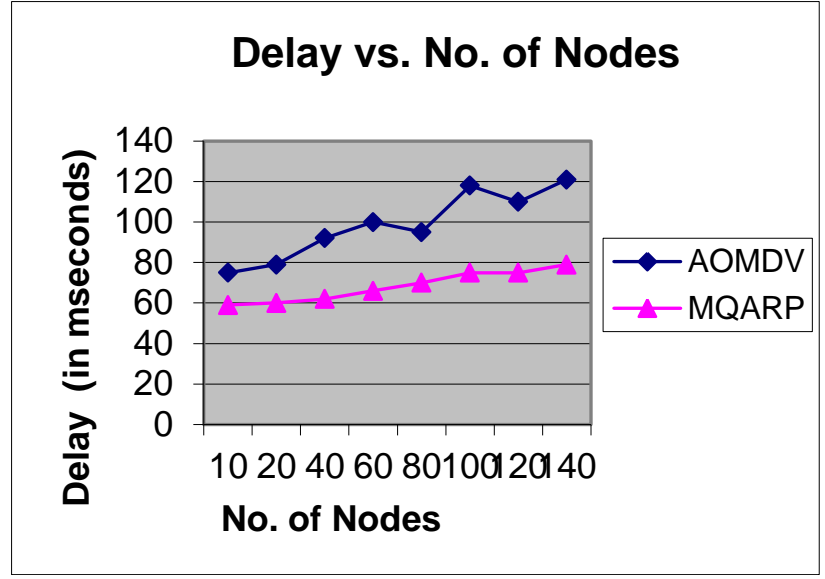

Figure 5 Delay versus No. of Nodes

From the figure 4 it can be observed that for the low pause time the delay is more. That means for high mobility the performance of the protocols in terms of the delay degrades. Similarly the figure 5 shows that as the number of nodes are increasing the performance in terms of delay degrades. But in both the cases the delay of the MQARP is showing a linear graph.

4.4.3 Jitter versus Mobility and Number of nodes

The Figure 6 shows the Jitter versus pause time for the number of nodes $=80$. And Figure 7 shows Delay versus Number of nodes for the Pause time $=10$ seconds

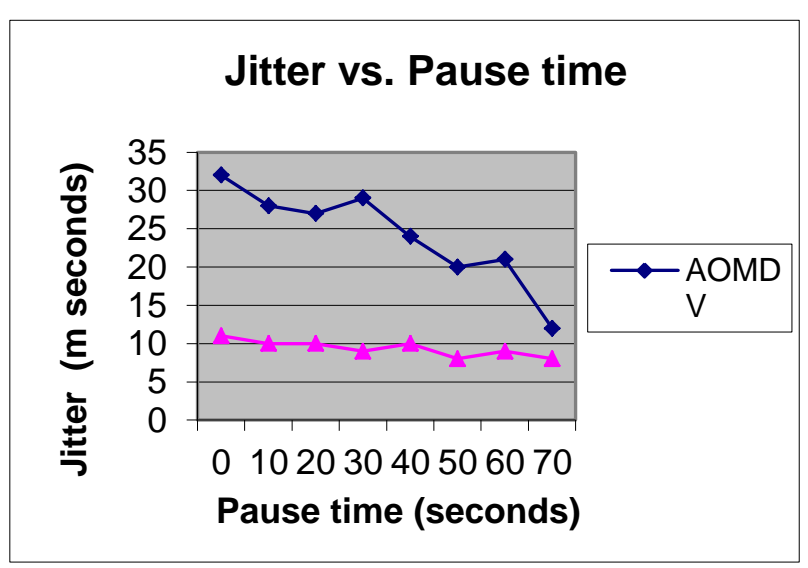

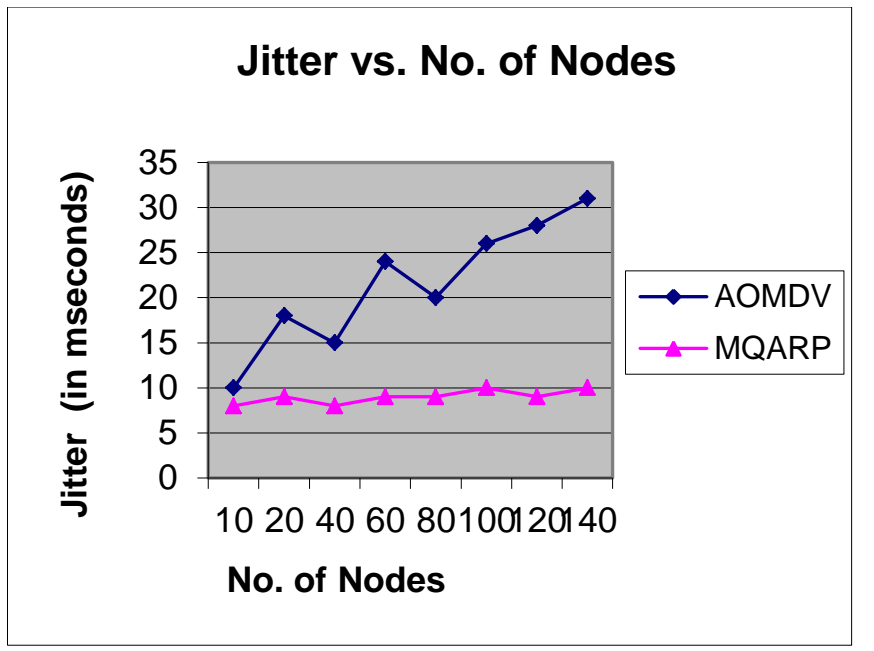

Figure 7 Jitter versus Number of nodes

From the figure 6 it can be observed that for the low pause time the Jitter is more. That means for high mobility the performance of the protocols in terms of the variation in delay degrades. Similarly the figure 7 shows that as the number of nodes are increasing the performance in terms of Jitter degrades. But in both the cases the Jitter of the MQARP is showing a linear graph with very less change in delay.

\section{ACKNOWLEDGMENTS}

We are thankful to the Manipal Institute of Technology, Manipal for providing an opportunity to do this work under this Institution.

\section{CONCLUSIONS AND FUTUREWORK}

The performance of the two MANET Routing Protocols by varying node density, speed and pause time is investigated in this work using NS-2.34. The performances of these two routing protocols show some differences in low, medium and high node densities. From the experimental analysis it is concluded that the AOMDV protocol can be used with MANET having low density with low mobility and high density with low mobility. The QoS metrics Throughput, Jitter and delay are almost same for both AOMDV and MQARP for this situation. For the MANET with medium and large density with high mobility, the performance of AOMDV in terms of Throughput, Jitter and delay degrades. But the performance of MQARP is improved for the MANET with high density, high mobility and high speed situations. The Jitter of MQARP is always linearly varying. So this new protocol is suitable for video and audio data transmission. In future the QoS can be further improved by including the energy constraint along with timestamp and the link life time.

\section{REFERENCES}

[1] Falko Dressler, "Self-Organization in Ad Hoc Networks: Overview and Classification", Autonomic Networking Group, Dept. of Computer Science 7,University of Erlangen, Martensstr 3, 91058 Erlangen, Germany, 2006.

Figure 6 Jitter versus Pause time 
[2] A text book titled "Adhoc Wireless Networks Architecture and Protocols", by C Siva Ram Murthy and B S Manoj, Pearson Education, 2005.

[3] S. Chakrabarti and A. Mishra, "Quality of Service Challenges for Wireless Mobile Ad Hoc Networks," International Journal of Wireless Communication and Mobile Computing., vol. 4, pp. 129-53, Mar 2004.

[4] Mahesh Marina, Samir Das , "Ad hoc on-demand multipath distance vector routing (AOMDV)", ACM digital library, 2002.

[5] Perkins, C.; Belding-Royer, E., Das S, Ad hoc OnDemand Distance Vector (AODV) Routing. IETF. RFC 3561. https://tools.ietf.org/html/rfc3561. July 2003.

[6] Shahram Jamali, Bita Safarzadeh, Hamed Alimohammadi, "A stable QoS aware reliable ondemand distance vector routing protocols for mobile Ad Hoc networks", Scientific Research and Essays Volume 6, Academic Journals, July 2011.

[7] S. Chakrabarti and A. Mishra, "Quality of Service Challenges for Wireless Mobile Ad Hoc Networks," International Journal of Wireless Communication and Mobile Computing, Volume 4, pp. 129-53, March 2004.
[8] J. Stine and G. de Veciana, "A Paradigm for Quality of Service in Wireless Ad Hoc Networks using Synchronous Signaling and Node States," IEEE JSAC, vol. 22, Sept. 2004

[9] Taejoon Park, Student Member, IEEE, and Kang G. Shin, Fellow, IEEE, "Optimal Tradeoffs for Location-Based Routing in Large-Scale Ad Hoc Networks, IEEE TRANSACTIONS ON NETWORKING, VOL. 13, NO. 2, APRIL 2005

[10] Ronald Beaubrun and BadjiMolo, "Using DSR for Routing multimedia traffic in MANETs", January 2010.

[11] Chandra MouliVenkataSrinivasAkana, Sandeep Kumar, Dr C Divakar "QoS for Real time transmission on MANETs", International Journal of Advanced Networkingand Applications volume: 02, Issue: 03, Pages: 679-685, 2010.

[12] The network simulator: ns-2[Online] Available: http:/www.isi.edu/nsnam/ns/index.html.

[13] Tutorial for network Simulator Available: http:/www.isi.edu/nsnam/ns/tutorial. 